\title{
Emerging Regenerative Approaches for Periodontal Reconstruction: A Consensus Report From the AAP Regeneration Workshop
}

\author{
David L. Cochran ${ }^{\star}$, Charles M. Cobb ${ }^{\dagger}$, Jill D. Bashutski ${ }^{\ddagger}$, Yong-Hee Patricia Chun ${ }^{\star}$, Zhao

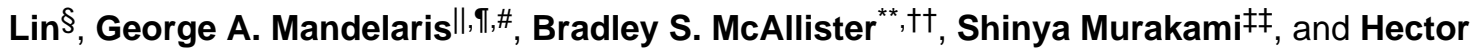 \\ F. Rios ${ }^{\ddagger}$ \\ *Department of Periodontics, University of Texas Health Science Center at San Antonio Dental \\ School, San Antonio, TX \\ tDepartment of Periodontics, School of Dentistry, University of Missouri-Kansas City, Kansas \\ City, MO \\ ‡Department of Periodontics and Oral Medicine, School of Dentistry, University of Michigan, Ann \\ Arbor, MI \\ $\S$ Department of Periodontics, Virginia Commonwealth University School of Dentistry, Richmond, \\ VA \\ IPrivate practice, Park Ridge and Oakbrook Terrace, IL \\ IDepartment of Oral and Maxillofacial Surgery, School of Dentistry, Louisiana State University, \\ New Orleans, LA \\ \#Department of Graduate Periodontics, College of Dentistry, University of Illinois at Chicago, \\ Chicago, IL \\ ${ }^{* *}$ Private practice, Tigard, OR \\ ${ }^{\dagger}$ Department of Periodontology, Oregon Health and Science University, Portland, OR \\ \#‡Department of Periodontology, Graduate School of Dentistry, Osaka University, Osaka, Japan
}

\section{Abstract}

Background-Historically, periodontal regeneration has focused predominantly on bone substitutes and/or barrier membrane application to provide for defect fill and/or selected cell repopulation of the lesion. More recently, a number of technologies have evolved that can be viewed as emerging therapeutic approaches for periodontal regeneration, and these technologies were considered in the review paper and by the consensus group. The goal of this consensus report on emerging regenerative approaches for periodontal hard and soft tissue reconstruction was to develop a consensus document based on the accompanying review paper and on additional materials submitted before and at the consensus group session.

Correspondence: Dr. David L. Cochran, Department of Periodontics, University of Texas Health Science Center at San Antonio Dental School, 7703 Floyd Curl Dr., San Antonio, TX 78240. Fax: 210/567-3393; cochran@uthscsa.edu. 
Methods-The review paper was sent to all the consensus group participants in advance of the consensus conference. In addition and also before the conference, individual consensus group members submitted additional material for consideration by the group. At the conference, each consensus group participant introduced themselves and provided disclosure of any potential conflicts of interest. The review paper was briefly presented by two of the authors and discussed by the consensus group. A discussion of each of the following topics then occurred based on the content of the review: a general summary of the topic, implications for patient-reported outcomes, and suggested research priorities for the future. As each topic was discussed based on the review article, supplemental information was then added that the consensus group agreed on. Last, an updated reference list was created.

Results-The application of protein and peptide therapy, cell-based therapy, genetic therapy, application of scaffolds, bone anabolics, and lasers were found to be emerging technologies for periodontal regeneration. Other approaches included the following: 1) therapies directed at the resolution of inflammation; 2) therapies that took into account the influence of the microbiome; 3 ) therapies involving the local regulation of phosphate and pyrophosphate metabolism; and 4) approaches directed at harnessing current therapies used for other purposes. The results indicate that, with most emerging technologies, the specific mechanisms of action are not well understood nor are the specific target cells identified. Patient-related outcomes were typically not addressed in the literature. Numerous recommendations can bemade for future research priorities for both basic science and clinical application of emerging therapies. The need to emphasize the importance of regeneration of a functional periodontal organ system was noted. The predictability and efficacy of outcomes, as well as safety concerns and the cost-to-benefit ratio were also identified as key factors for emerging technologies.

Conclusions-A number of technologies appear viable as emerging regenerative approaches for periodontal hard and soft tissue regeneration and are expanding the potential of reconstructing the entire periodontal organ system. The cost-to-benefit ratio and safety issues are important considerations for any new emerging therapies.

Clinical Recommendation-At this time, there is insufficient evidence on emerging periodontal regenerative technologies to warrant definitive clinical recommendations.

\section{Keywords}

Alveolar bone grafting; bonematrix; guided tissue regeneration; periodontal; periodontitis; tissue engineering

Members of the Emerging Regenerative Approaches for Periodontal Reconstruction consensus group met and began with individual introductions and provision of appropriate disclosures. The authors of the written review provided a summary of the construction and contents of the review paper. ${ }^{1}$ Each member of this consensus group provided comments on the review.

As an overview, one of the issues arising was the question of defining what constitutes an emerging technology. The group considered two broad categories: 1) products and components of products approved by the US Food and Drug Administration (FDA) and 2) non-approved therapeutic modalities. For example, components of FDA-approved products 
are being examined for periodontal regeneration and thus were considered an emerging therapeutic approach. In addition, currently available therapeutics that have limited data supporting their use in periodontal regeneration were also considered an emerging technology.

We discussed the contents of the review paper and made suggestions for additions. The consensus group agreed with the contents of the review paper and the scope of products and technologies that were covered in the paper with the additions and comments noted below.

Most of the emerging approaches discussed in the review paper were focused on the concepts of tissue engineering ${ }^{2}$ and also included other approaches. The topics discussed included the following: 1 ) protein and peptide therapy; ${ }^{3-8}$ 2) cell-based therapy; ${ }^{9}$ 3) genetic therapy; 4) scaffolds; ${ }^{10}$ 5) bone anabolics; and 6) lasers. FDA-approved products evaluated included the following: 1) enamel matrix derivative; 2 ) recombinant human platelet-derived growth factor; and 3) anorganic bone matrix. Non-approved therapeutic modalities included the following: 1) recombinant human fibroblast growth factor-2; 2) recombinant human growth differentiation factor-5; 3) bone morphogenetic proteins (BMP-2, BMP-7, BMP-6, andBMP-12); 4) parathyroid hormone/ teriparatide; 5) brain-derived neurotrophic factor; and 6) sclerostin antibodies. ${ }^{11}$ Cell-based therapies included the following: 1) mesenchymal stem cells; 2) bone marrow stromal cells; 3) periodontal ligament cells; 4) embryonic stem cells; and 5) induced pluripotent stem cells. Gene therapies included viral and non-viral vectors. Scaffolds are also promising for delivery of growth factors and gene therapy and may be composed of either natural or synthetic polymeric materials. ${ }^{12,13}$ Finally, various types of lasers were discussed.

In addition to the approaches highlighted in the review, the consensus group recognized that many of the therapeutics in current use are based on fundamental knowledge and understanding of the development of the periodontium. ${ }^{14-16}$ Furthermore, the host inflammatory response should also be considered, as well as such interactions between the host genome, epigenetics, and the microbiome. ${ }^{17-20}$ Areas of future interest might include inflammatory regulators such as resolvins ${ }^{21}$ and interleukin-17 antibodies, as well as phosphate/pyrophosphate local regulation. ${ }^{22}$

\section{IMPLICATIONS OF REVIEW TO PATIENTRELATED CLINICAL OUTCOMES}

The review did not identify any reports addressing patient-reported outcomes. However, the review did reveal clinical parameter-based outcomes of several individualized approaches over various time periods, with the longest follow-up being 3 years in one report of 83 patients. ${ }^{23}$ Although there are multiple papers focused on various emerging technologies, there are no studies that allow for direct comparison of clinical outcomes.

When dealing with emerging technologies, there can be both positive and negative issues of clinician adoption and patient acceptance of treatment.

Barriers to adopting this technology include limited evidence supporting efficacy and indications for use. With emerging technologies, safety issues include unknown long-term effects along with known risk for rare but serious side effects, such as sarcoma. This is, in 
part, attributable to evaluation of such emerging technologies in populations defined by selected inclusion/exclusion criteria, further limiting outcome assessments.

With any emerging technology, the cost-to-benefit ratio for clinicians and patients must be determined. For the clinician, peer and market pressures, surgical time, technical complexity, healing times, predictability, liability, and cost must be considered.

For patients, experience of pain and morbidity, adverse events, both short and long term, cost, time, material (ethical and religious concerns), esthetic perceptions, and satisfaction with treatment outcomes should be considered.

\section{RESEARCH PRIORITIES FOR THE FUTURE}

With all emerging therapies, the prevalence, predictability, and efficacy of outcomes and safety should be well defined. Future research should promote the goal of emerging technologies to regenerate the periodontium as a functional organ system. The review made a number of recommendations, and the consensus group highlighted that future studies should do the following: 1) develop a non-invasive assessment of clinical periodontal regeneration; 2) evaluate the efficacy and safety of combining emerging and/or current therapies; 3) validate existing and/or emerging therapies being used "off label"; 4) explore therapies developed for other purposes for their application to periodontal regeneration; 5) define the individual's genetic and epigenetic profile so that it can be used to personalize the choice of therapy; 6) assess the effect of individual disease pathogenesis, etiology, and healing potential on therapeutic treatment selection; 7) optimize the understanding of risk factors to aid in the selection of appropriate therapy and the achievement of enhanced outcomes to restore the structure and function of the periodontium; 8) define molecular and cellular mechanisms of the emerging therapy using in vitro and in vivo models; 9) identify developmental pathways of the periodontium for potential application in regenerative therapy; 10) focus on developing minimally invasive technologies to minimize pain and morbidity without compromising outcomes; 11) define what constitutes clinical success; and 12) characterize the effect of the selected therapy on the patient's quality of life.

\section{Acknowledgments}

Dr. Cochran has received research funding and consulting fees from Sunstar Americas (Chicago, Illinois). Dr. Cobb has received research funding from OraPharma (Horsham, Pennsylvania) and is an unpaid consultant for Hu-Friedy (Chicago, Illinois) and Livionex (Los Gatos, California). Dr. Bashutski has received research funding from Eli Lilly (Indianapolis, Indiana), Biomet 3i (Palm Beach Gardens, Florida), and Osteology Foundation (Lucerne,

Switzerland). Research by Dr. Chun was supported by the National Institutes of Health/National Institute of Dental and Craniofacial Research (K08DE022800) and the National Center for Advancing Translational Sciences (UL1TR001120). Dr. McAllister has received research funding and consulting, advisor, and lecture fees from ACE Surgical Supply (Brockton, Massachusetts), Nobel Biocare (Zürich, Switzerland), Institute Straumann (Basel, Switzerland), Osteohealth (Shirley, New York), Organogenesis (Canton, Massachusetts), and Cytograft Tissue Engineering (Novato, California) as well as advisor fees from Imaging Sciences International (Hatfield, Pennsylvania). Dr. Murakami has received research funding and consulting fees from Kaken Pharmaceutical (Tokyo, Japan). Dr. Rios has received research funding from Osteology Foundation, has served as a consultant for Geitslich Pharma (Wolhusen, Switzerland) and Organogenesis, and his research has been supported by the National Institutes of Health/National Institute of Dental and Craniofacial Research (1K23DE019872, R21DE023845-01A1, and R56DE022787-01A1). Drs. Lin and Mandelaris report no conflicts of interest related to this consensus report. The 2014 Regeneration Workshop was hosted by the American Academy of Periodontology (AAP) and supported in part by the AAP Foundation, Geistlich Pharma North America, Colgate-Palmolive, and the Osteology Foundation. 


\section{References}

1. Lin Z, Rios HF, Cochran DL. Emerging regenerative approaches for periodontal reconstruction: A systematic review from the AAP regeneration workshop. J Periodontol. 2015; 86(Suppl):S134S152. [PubMed: 25644297]

2. Somerman M. Growth factors and periodontal engineering: Where next? J Dent Res. 2011; 90:7-8. [PubMed: 21041551]

3. Amin HD, Olsen I, Knowles J, Dard M, Donos N. Atyrosine-rich amelogenin peptide promotes neovasculogenesis in vitro and ex vivo. Acta Biomater. 2014; 10:1930-1939. [PubMed: 24321350]

4. Amin HD, Olsen I, Knowles JC, Donos N. Differential effect of amelogenin peptides on osteogenic differentiation in vitro: Identification of possible new drugs for bone repair and regeneration. Tissue Eng Part A. 2012; 18:1193-1202. [PubMed: 22320389]

5. Foster BL, Nagatomo KJ, Nociti FH Jr, et al. Central role of pyrophosphate in acellular cementum formation. PLoS One. 2012; 7:e38393. [PubMed: 22675556]

6. Iizuka S, Kudo Y, Yoshida M, et al. Ameloblastin regulates osteogenic differentiation by inhibiting Src kinase via cross talk between integrin beta1 and CD63. Mol Cell Biol. 2011; 31:783-792. [PubMed: 21149578]

7. Kakegawa A, Oida S, Gomi K, et al. Cytodifferentiation activity of synthetic human enamel sheath protein peptides. J Periodontal Res. 2010; 45:643-649. [PubMed: 20572923]

8. Kanazashi M, Gomi K, Nagano T, Tanabe T, Arai T, Fukae M. The 17-kDa sheath protein in enamel proteins induces cementum regeneration in experimental cavities created in a buccal dehiscence model of dogs. J Periodontal Res. 2006; 41:193-199. [PubMed: 16677288]

9. Arany PR, Cho A, Hunt TD, et al. Photoactivation of endogenous latent transforming growth factorbeta1 directs dental stem cell differentiation for regeneration. Sci Transl Med. 2014; 6:238ra69.

10. Costa PF, Vaquette C, Zhang Q, Reis RL, Ivanovski S, Hutmacher DW. Advanced tissue engineering scaffold design for regeneration of the complex hierarchical periodontal structure. $\mathrm{J}$ Clin Periodontol. 2014; 41:283-294. [PubMed: 24304192]

11. Das S, Sakthiswary R. Bone metabolism and histomorphometric changes in murine models treated with sclerostin antibody: A systematic review. Curr Drug Targets. 2013; 14:1667-1674. [PubMed: 24354585]

12. Oortgiesen DA, Walboomers XF, Bronckers AL, Meijer GJ, Jansen JA. Periodontal regeneration using an injectable bone cement combined with BMP-2 or FGF-2. J Tissue Eng Regen Med. 2014; 8:202-209. [PubMed: 22552898]

13. Yamano S, Haku K, Yamanaka T, et al. The effect of a bioactive collagen membrane releasing PDGF orGDF-5 on bone regeneration. Biomaterials. 2014; 35:2446-2453. [PubMed: 24388383]

14. Cortellini P, Pini-Prato G, Nieri M, Tonetti MS. Minimally invasive surgical technique and enamel matrix derivative in intrabony defects: 2 . Factors associated with healing outcomes. Int $\mathbf{J}$ Periodontics Restorative Dent. 2009; 29:257-265. [PubMed: 19537465]

15. Fukae M, Kanazashi M, Nagano T, Tanabe T, Oida S, Gomi K. Porcine sheath proteins show periodontal ligament regeneration activity. Eur J Oral Sci. 2006; 114(Suppl 1):212-218. discussion 254-256, 381-382. [PubMed: 16674688]

16. Warotayanont R, Frenkel B, Snead ML, Zhou Y. Leucine-rich amelogenin peptide induces osteogenesis by activation of the Wnt pathway. Biochem Biophys Res Commun. 2009; 387:558563. [PubMed: 19615979]

17. Hajishengallis G. Aging and its impact on innate immunity and inflammation: Implications for periodontitis. J Oral Biosci. 2014; 56:30-37. [PubMed: 24707191]

18. Hajishengallis G, Sahingur SE. Novel inflammatory pathways in periodontitis. Adv Dent Res. 2014; 26:23-29. [PubMed: 24736701]

19. Maekawa T, Abe T, Hajishengallis E, et al. Genetic and intervention studies implicating complement C3 as a major target for the treatment of periodontitis. J Immunol. 2014; 192:6020 6027. [PubMed: 24808362]

20. Moutsopoulos NM, Konkel J, Sarmadi M, et al. Defective neutrophil recruitment in leukocyte adhesion deficiency type I disease causes local IL-17-driven inflammatory bone loss. Sci Transl Med. 2014; 6:229ra40. 
21. Mustafa M, Zarrough A, Bolstad AI, et al. Resolvin D1 protects periodontal ligament. Am J Physiol Cell Physiol. 2013; 305:C673-C679. [PubMed: 23864609]

22. Rodrigues TL, Nagatomo KJ, Foster BL, Nociti FH, Somerman MJ. Modulation of phosphate/ pyrophosphate metabolism to regenerate the periodontium: A novel in vivo approach. $\mathbf{J}$ Periodontol. 2011; 82:1757-1766. [PubMed: 21488756]

23. Nevins M, Kao RT, McGuire MK, et al. Platelet-derived growth factor promotes periodontal regeneration in localized osseous defects: 36-month extension results from a randomized, controlled, double-masked clinical trial. J Periodontol. 2013; 84:456-464. [PubMed: 22612364] 